Analyses are based on data from six studies of child and adolescent development, health and adaptation; The Study of Early Child Care and Youth Development (NICHD; N=1009); The Youth Risk Behaviour Survey (YRBS; N=14 041); The National Longitudinal Study of Adolescent Health (AddHealth; $N=20$ 745), The Youth Violence Survey $(\mathrm{N}=4116)$; the National Study of Child and Adolescent Well-Being ( $N=6231)$, and the Behavioural Health/Juvenile Justice Project (BHJJ) ( $\mathrm{N}=455)$. These six studies capture samples of normative adolescents as well as a sample of high-risk youth defined as youth who have been processed through the Child Welfare System and a sample of youth who have been adjudicated. Our findings show that the prevalence of suicide ideation varied from $7.0 \%$ to $23.8 \%$ among adolescents 15 years of age and was highest among adolescents in high-risk samples. For example, in the BHJJ a sample of adjudicated youth, at the time of intake $44 \%$ of participants had talked about committing suicide and $17 \%$ had previously attempted suicide. In comparison, in the nationally representative Add Health, $3.6 \%$ of participants had attempted suicide. Prevalence of suicidal behaviours as well as the characteristics of those most vulnerable to suicidal behaviours varies across sociocultural contexts. These variations need to be considered in future research and in the design and implementation of prevention and intervention efforts.

\section{EPIDEMIOLOGY OF SUICIDAL BEHAVIOUR AMONG YOUTH IN THE U.S.: EXAMINING THE INFLUENCE OF SOCIOCULTURAL CONTEXT}

M H Swahn*, R M Bossarte, M Van Dulmen, J Kretschmar, A Jones Correspondence: Georgia State University, P.O. Box 3995, Atlanta, GA 30302, USA

10.1136/ip.2010.029215.945

The objectives of this paper are to determine the prevalence, demographic characteristics and patterns of suicidal behaviours among youth across sociocultural contexts in the U.S. 